\title{
Production of Oxidative and Hydrolytic Enzymes by Coprinus cinereus (Schaeff.) Gray from Sisal Wastes Supplemented with Cow Dung Manure
}

\author{
Prosper Raymond, Anthony Manoni Mshandete, and Amelia Kajumulo Kivaisi \\ Department of Molecular Biology and Biotechnology, College of Natural and Applied Sciences, University of Dar es Salaam, \\ Uvumbuzi Road, P.O. Box 35179, Dar es Salaam, Tanzania
}

Correspondence should be addressed to Prosper Raymond; promosha@yahoo.com

Received 8 September 2015; Accepted 15 October 2015

Academic Editor: Triantafyllos Roukas

Copyright (C) 2015 Prosper Raymond et al. This is an open access article distributed under the Creative Commons Attribution License, which permits unrestricted use, distribution, and reproduction in any medium, provided the original work is properly cited.

\begin{abstract}
The activity of oxidative and hydrolytic enzymes of the edible and medicinal white rot fungi Coprinus cinereus (Schaeff.) Gray mushroom was observed during mycelia growth and fruiting body development in solid substrate fermentation using sisal waste fractions amended with cow dung manure as supplement. Laccase had the highest titre value among the five detected enzymes. Its activity was higher during mycelia growth compared to fruiting phase, with $10 \%$ supplemented substrate formulation unmixed sisal leaf decortication residues [abbreviated SL: SB (100:0)] displaying the highest activity of $39.45 \pm 12.05 \mathrm{Ug}^{-1}$. Lignin peroxidase (LiP) exhibited a characteristic wave-like pattern with the highest peaks found either during full mycelia colonization or soon after first flush harvest; the highest activity of $1.93 \pm 0.62 \mathrm{Ug}^{-1}$ was observed on unsupplemented SL : SB (100:0) substrate formulation during mycelia colonization. For hydrolytic enzymes, the highest carboxymethyl cellulase (CMCase) activity of $2.03 \pm 0.70 \mathrm{Ug}^{-1}$ was observed on $20 \%$ supplemented SL: SB $(0: 100)$ after first flush; that of pectinase $\left(1.90 \pm 0.32 \mathrm{Ug}^{-1}\right)$ was revealed after third flush on $10 \%$ supplemented SL: SB $(0: 100)$ substrate formulation while $10 \%$ supplemented SL:SB $(25: 75)$ exhibited the highest xylanase activity $\left(1.23 \pm 0.12 \mathrm{Ug}^{-1}\right)$ after first flush. These findings show that the activities of both oxidative and hydrolytic enzymes were regulated in line with developmental phase of growth of Coprinus cinereus.
\end{abstract}

\section{Introduction}

Enormous quantities of organic wastes from agricultural and food processing industries which are lignocellulosic in nature are generated annually [1]. A large proportion of these wastes are dumped or left to rot near the factories, burnt, or shredded and composted and used for landfill or improving soil quality [2]. However, with the application of appropriate bioconversion technology, these wastes represent a valuable resource especially for areas with agriculturalbased economies.

Edible mushroom cultivation represents one of the most economically viable processes for the bioconversion of these lignocellulosic wastes which include diverse materials such as cereal straws, bagasse, sawdust, cotton wastes, banana leaves, coffee grounds, and sisal wastes [3-5]. Utilization of insoluble lignocellulosic substrates by these fungi depends on their capability to synthesize the relevant hydrolytic (cellulases and hemicellulases) and oxidative (ligninolytic) extracellular enzymes [6]. These are responsible for the degradation of substrate major components, that is, cellulose, hemicellulose, and lignin, into low molecular weight compounds that can be assimilated for fungal nutrition [7]. Production of these enzymes by the fungal mycelium is a crucial part of the colonization process and an important determinant of mushroom growth, development, and yields $[8,9]$. Consequently, it is important to evaluate basidiomycetes hydrolytic and oxidative enzymes activity while cultivating in the presence of lignocellulose since in lignified plant substrates cellulose, hemicelluloses, and lignin are linked intra- and intermolecularly [6].

In Tanzania, high quantities of solid sisal wastes are produced annually as only $2 \%$ of the plants are currently used for sisal fibre production while the remaining $98 \%$ 
are commonly disposed of in the raw into the environment [10-12]. Although their use in the general cultivation of Coprinus cinereus has been established $[4,5]$, no information exists regarding enzymatic activity, function, and degradative capacity of Coprinus when cultivated upon this substrate. The aim of this work was, therefore, to determine the profile of the oxidative and hydrolytic enzymes during vegetative growth, fruiting, and postharvest stage in three flushes of Coprinus cinereus on solid sisal waste fractions supplemented with cow manure.

\section{Material and Methods}

2.1. Fungal Strain, Tissue Culture, and Spawn Preparation. Coprinus cinereus (Schaeff.) Gray was collected from sisal decortications waste dumpsite at Kidugalo sisal estate, Morogoro, Tanzania. These mushrooms were brought to the laboratory on the same day for tissue culture. The mycelia from living mushroom fruit bodies were aseptically obtained following the tissue culture protocol according to Dhouib et al. [13]. Spawn were prepared with intact sorghum grains bought from Kariakoo market, Dar es Salaam, according to Mshandete and Cuff [4].

2.2. Substrate Preparation and Mushroom Cultivation Experiments. Fresh sisal leaf decortication residues, a leafy biomass produced during sisal decortications and sisal boles, were obtained from Kidugalo sisal decortications factory at Morogoro region, Tanzania. These substrates were further pretreated prior to Coprinus cinereus cultivation as reported in Raymond et al. [5]. The substrates for mushroom cultivation consisted of composted sisal leaf (SL) decortication residues and sisal boles (SB) formulated in proportions of $(100: 0)$, $(75: 25),(50: 50),(25: 75)$, and $(0: 100)$ and supplemented with cow manure at the rate of $10 \%, 20 \%$, and $30 \%$ on dry weight basis. Cultivation was carried out in solid state fermentation bioreactors (SSFBs) which consisted of 3-litre rectangular plastic containers with dimension of $23 \mathrm{~cm} \times 14 \mathrm{~cm} \times$ $9 \mathrm{~cm}$ (length, width, and height, resp.) (Cello Domestic Ware (Mkate), Dar es Salaam, Tanzania). A total of 136 aeration holes of $0.7 \mathrm{~cm}$ in diameter and $3 \mathrm{~cm}$ apart were made in all the sides of the bioreactor to facilitate aeration during spawn running. All substrates were inoculated with fully colonized 14-day old spawn at 6\% (wet weight spawn/wet weight substrate) under aseptic conditions. The conditions during spawn running in the room were $28 \pm 2^{\circ} \mathrm{C}$ and relative air humidity of $78 \pm 2 \%$. Once the mycelia of $C$. cinereus strain had grown throughout the whole substrate, the bioreactors were removed and transferred to a fruiting room, where the environment was illuminated by sunlight. Relative humidity in the room was increased to $86 \pm 4 \%$ while temperature was decreased to $26.5 \pm 0.05^{\circ} \mathrm{C}$, respectively, by pouring 25 litres of cold water and ice cubes per day on the floor. The mushroom fruiting bodies were harvested according to Mshandete and Cuff [4].

2.3. Extraction of Crude Enzymes from Substrates. Substrate samples with active mycelia of about $20 \mathrm{~g}( \pm 2 \mathrm{~g})$ were taken from the container at different intervals starting on the 5th day, 10th day (full colonization), 15th day (first flush), 20th day (second flush), and 25th day (third flush). These samples were weighed and mixed with $50 \mathrm{~mL}$ of the subsequent extraction buffer and homogenized. The mixture was agitated on orbital shaker (Edmund Bühler, 7400 Tübingen, Germany) for one hour at $120 \mathrm{rpm}$. During this step, all flasks containing the substrate-buffer mixture were kept on ice throughout the entire shaking period. These mixtures were then filtered through double layered cheesecloth followed by centrifugation at $6000 \mathrm{rpm}$ for 10 minutes (Mikro 22R centrifuge, Hettich, England) at $4^{\circ} \mathrm{C}$ to remove residual particles. The clear supernatants obtained were regarded as crude enzymes which were later used for enzyme activity assays.

2.4. Enzyme Assays. Laccase production in the crude enzyme extract was determined according to Risdianto et al. [14] by monitoring the absorbance change at $420 \mathrm{~nm}\left(A_{420 \mathrm{~nm}}\right)$ related to the rate of oxidation of $1 \mathrm{mM} \mathrm{2,2}$-azino-bis-[3ethylbenzthiazoline-6-sulfonate] (ABTS) in $50 \mathrm{mM}$ sodium acetate buffer ( $\mathrm{pH}$ 5.5). Assays were performed in $1.5 \mathrm{~mL}$ cuvette containing $0.5 \mathrm{mM}$ of ABTS in $50 \mathrm{mM}$ sodium acetate buffer ( $\mathrm{pH} 5.5)$ and $50 \mu \mathrm{L}$ of crude enzyme [15]. The oxidation of ABTS was monitored by the formation of intense blue-green color which was observed by measuring the increase in the absorbance at $420 \mathrm{~nm}$, with a molar extinction coefficient of $36,000 \mathrm{M}^{-1} \mathrm{~cm}^{-1}$ using UV-Visible spectrophotometer (Jenway, Genova, Bibby Scientific Ltd., England). One unit of activity was defined as the amount of enzyme which leads to the oxidation of $1 \mu \mathrm{mol}$ of ABTS per minute.

Lignin peroxidase activity was determined at $30^{\circ} \mathrm{C}$ using the method of Sugiura et al. [16] in a reaction mixture $(1.5 \mathrm{~mL})$ containing $20 \mathrm{mM}$ citrate buffer ( $\mathrm{pH} 3.0), 10 \mathrm{mM}$ veratryl alcohol (=3,4-dimethoxybenzyl alcohol), and $0.1 \mathrm{~mL}$ crude enzyme extract. The reaction was started by adding $10 \mathrm{mM} \mathrm{H}_{2} \mathrm{O}_{2}$ in the assay mixture after incubation at $30^{\circ} \mathrm{C}$ for 5 minutes, and the increase in absorbance was followed spectrophotometrically using Jenway UV-Visible spectrophotometer (Bibby Scientific Ltd., United Kingdom) at $310 \mathrm{~nm}$ (extinction coefficient, $\varepsilon 310=9300 \mathrm{M}^{-1} \mathrm{~cm}^{-1}$, ) due to oxidation of veratryl alcohol to veratraldehyde $(=3,4$ dimethoxybenzaldehyde). One unit ( $1 \mathrm{U}$ ) of LiP activity was defined as the amount of enzyme that oxidized $1.0 \mu$ mole of veratryl alcohol per minute at $\mathrm{pH} 3$ and $30^{\circ} \mathrm{C}$.

Carboxymethyl cellulase (CMCase), pectinase, and xylanase activities were estimated using the DNS method according to Miller [17], measuring the amount of reducing sugars (as glucose for CMCase/pectinase or xylose in case of xylanase) liberated in reaction mixtures containing $0.5 \%$ carboxymethyl cellulose (Sigma), 0.5\% pectin (citrus pectin, Sigma), or $0.5 \%$ birch wood xylan (Sigma) and supernatant, respectively. The incubation times were 30 and 60 minutes at $30^{\circ} \mathrm{C}$, respectively [18]. Standard curves were obtained using glucose or xylose. Enzyme activities (U) were expressed as the amount of enzyme releasing $1 \mu$ mole of glucose or xylose equivalent per $\mathrm{mL}$ per min. All enzyme assays were carried out in triplicate. 
TABLE 1: Days for completion of spawn running and pinheads and fruiting bodies formation.

\begin{tabular}{|c|c|c|c|c|}
\hline Supplement level & Substrate formulation & $\begin{array}{l}\text { Days for completion of } \\
\text { spawn run }\end{array}$ & $\begin{array}{l}\text { Days for pinheads } \\
\text { formation }\end{array}$ & $\begin{array}{l}\text { Days for fruiting } \\
\text { bodies formation }\end{array}$ \\
\hline \multirow{5}{*}{$0 \%$} & SL : SB (100:0) & $12 \pm 1$ & $15 \pm 1$ & $17 \pm 1$ \\
\hline & SL: SB $(75: 25)$ & $11 \pm 1$ & $13 \pm 1$ & $15 \pm 3$ \\
\hline & SL : SB $(50: 50)$ & $10 \pm 1$ & $11 \pm 1$ & $13 \pm 2$ \\
\hline & SL: SB $(25: 75)$ & $10 \pm 1$ & $12 \pm 1$ & $13 \pm 1$ \\
\hline & SL : SB $(0: 100)$ & $13 \pm 2$ & $14 \pm 1$ & $16 \pm 1$ \\
\hline \multirow{5}{*}{$10 \%$} & SL : SB $(100: 0)$ & $11 \pm 1$ & $13 \pm 1$ & $15 \pm 2$ \\
\hline & SL : SB $(75: 25)$ & $11 \pm 1$ & $14 \pm 1$ & $15 \pm 1$ \\
\hline & SL : SB $(50: 50)$ & $10 \pm 1$ & $12 \pm 1$ & $13 \pm 1$ \\
\hline & SL : SB $(25: 75)$ & $9 \pm 1$ & $10 \pm 1$ & $12 \pm 2$ \\
\hline & SL: SB $(0: 100)$ & $12 \pm 1$ & $13 \pm 1$ & $14 \pm 1$ \\
\hline \multirow{5}{*}{$20 \%$} & SL: SB $(100: 0)$ & $11 \pm 1$ & $14 \pm 1$ & $15 \pm 1$ \\
\hline & SL: SB $(75: 25)$ & $10 \pm 1$ & $15 \pm 1$ & $17 \pm 2$ \\
\hline & SL : SB $(50: 50)$ & $8 \pm 1$ & $10 \pm 1$ & $11 \pm 1$ \\
\hline & SL : SB $(25: 75)$ & $9 \pm 1$ & $11 \pm 1$ & $12 \pm 1$ \\
\hline & SL : SB $(0: 100)$ & $11 \pm 1$ & $12 \pm 1$ & $14 \pm 1$ \\
\hline \multirow{5}{*}{$30 \%$} & SL : SB $(100: 0)$ & $10 \pm 1$ & $12 \pm 0$ & $13 \pm 1$ \\
\hline & SL: SB $(75: 25)$ & $9 \pm 1$ & $12 \pm 1$ & $13 \pm 1$ \\
\hline & SL : SB $(50: 50)$ & $10 \pm 1$ & $12 \pm 1$ & $13 \pm 2$ \\
\hline & SL: SB $(25: 75)$ & $8 \pm 1$ & $9 \pm 1$ & $11 \pm 1$ \\
\hline & SL : SB $(0: 100)$ & $12 \pm 1$ & $14 \pm 1$ & $15 \pm 1$ \\
\hline
\end{tabular}

\section{Results}

3.1. Spawn Run and Pinhead and Fruiting Bodies Formation. The first well visible signs of fungal growth in solid state fermentation were seen two days after inoculation with Coprinus cinereus while the total vegetative growth (spawn running) on solid sisal waste fractions took an average of about $10 \pm 2$ days regardless of the supplementation rate used. The pinheads (primordia) appeared after $12 \pm 3$ days while it took 1-2 days for mature mushrooms to be ready for harvesting (Table 1). Three flushes of fruiting were obtained and the fruiting bodies in the first flushes weighed more than the fruiting bodies in subsequent flushes. This study revealed that different substrates showed varying yields and biological efficiencies with or without supplements. Results regarding mushroom yields, mushroom size, and biological efficiencies of these substrate formulations are reported elsewhere [5].

\subsection{Enzyme Profile during Solid State Cultivation of Coprinus cinereus on Sisal Waste Formulations}

3.2.1. Laccase Activities ( $U g^{-1} S M S$ Wet Wt.). Results of laccase activity profiles during solid state cultivation of $C$. cinereus on various solid sisal waste formulations supplemented with cow manure are presented in Table 2. Activity levels in all substrate formulations were found to increase during vegetative growth phase up to when the substrates were full colonized (day 10), with $10 \%$ supplemented substrate formulation SL:SB (100:0) displaying the highest activity peak of $39.45 \pm 12.05 \mathrm{Ug}^{-1}$ at this stage. However, there was abrupt decline of these activities soon after the first harvest in almost all substrate formulations except for some on which activities were found to increase again after the third flush harvest.

3.2.2. Lignin Peroxidase ( $U^{-1}$ SMS Wet Wt.). The lignin peroxidase (LiP) activities from early colonization till fruiting bodies development were fluctuating with a wave-like pattern (Table 3). The peak activity occurred twice between the full mycelia colonization and the third flush harvest. The highest peaks were found to be either during full mycelia colonization or just after first flush harvest. The highest activity of $1.93 \pm$ $0.62 \mathrm{Ug}^{-1}$ was observed on unsupplemented SL : SB (100:0) substrate formulation during full mycelia colonization.

3.2.3. CMCase Profile ( $U g^{-1}$ SMS Wet Wt.). The data in Table 4 indicate that the $C$. cinereus CMCase activity varied with different substrate formulation used. Higher activities were detected either during full mycelia colonization or soon after first flush harvest followed by gradual decline in the subsequent flushes. The highest activity of $2.03 \pm 0.70 \mathrm{Ug}^{-1}$ was observed on $20 \%$ supplemented SL : SB $(0: 100)$ after the first flush harvest.

3.2.4. Pectinase $\left(\mathrm{Ug}^{-1} \mathrm{SMS}\right.$ Wet Wt.). The pectinase activities were observed to increase gradually over days of cultivation (Table 5). Higher activities were found on spent substrates of 
TABLE 2: Laccase activity ( $\mathrm{Ug}^{-1}$ Spent Mushroom Substrate (SMS) wet weight) profiles of Coprinus cinereus at different stages during cultivation on solid sisal wastes supplemented with cow dung manure.

\begin{tabular}{|c|c|c|c|c|c|c|}
\hline \multirow{2}{*}{ Supplement level } & \multirow{2}{*}{ Days of incubation } & \multicolumn{5}{|c|}{ Substrate formulations } \\
\hline & & SL : SB (100:0) & SL : SB $(25: 75)$ & SL: SB (50:50) & SL: SB $(75: 25)$ & SL : SB $(0: 100)$ \\
\hline \multirow{5}{*}{$0 \%$} & 5 & $6.87 \pm 1.85$ & $9.56 \pm 2.85$ & $8.29 \pm 2.05$ & $5.09 \pm 1.23$ & $11.04 \pm 1.05$ \\
\hline & 10 & $34.87 \pm 5.73$ & $27.89 \pm 10.56$ & $20.05 \pm 4.90$ & $26.97 \pm 8.92$ & $24.67 \pm 6.43$ \\
\hline & 15 & $18.06 \pm 7.95$ & $24.95 \pm 7.50$ & $23.93 \pm 3.17$ & $24.12 \pm 1.03$ & $17.23 \pm 4.19$ \\
\hline & 20 & $15.82 \pm 2.20$ & $12.05 \pm 2.19$ & $13.89 \pm 1.94$ & $19.37 \pm 3.15$ & $6.52 \pm 1.73$ \\
\hline & 25 & $27.68 \pm 5.50$ & $2.97 \pm 1.12$ & $9.56 \pm 1.06$ & $2.98 \pm 0.87$ & $13.98 \pm 2.11$ \\
\hline \multirow{5}{*}{$10 \%$} & 5 & $3.09 \pm 1.02$ & $7.85 \pm 1.98$ & $9.86 \pm 3.11$ & $11.93 \pm 3.13$ & $6.93 \pm 2.12$ \\
\hline & 10 & $39.45 \pm 12.05$ & $29.73 \pm 4.83$ & $26.17 \pm 1.85$ & $29.28 \pm 7.93$ & $26.58 \pm 10.45$ \\
\hline & 15 & $28.89 \pm 8.72$ & $20.67 \pm 3.51$ & $17.83 \pm 2.54$ & $26.91 \pm 4.82$ & $24.91 \pm 3.19$ \\
\hline & 20 & $10.34 \pm 2.87$ & $16.84 \pm 4.97$ & $4.04 \pm 1.13$ & $22.36 \pm 3.12$ & $17.48 \pm 1.03$ \\
\hline & 25 & $8.55 \pm 3.89$ & $4.86 \pm 2.67$ & $12.14 \pm 2.19$ & $7.05 \pm 4.92$ & $9.87 \pm 2.10$ \\
\hline \multirow{5}{*}{$20 \%$} & 5 & $12.04 \pm 2.87$ & $9.69 \pm 2.65$ & $10.73 \pm 1.32$ & $7.93 \pm 1.49$ & $14.93 \pm 3.01$ \\
\hline & 10 & $28.67 \pm 10.55$ & $25.09 \pm 1.86$ & $27.85 \pm 7.57$ & $27.38 \pm 6.74$ & $28.27 \pm 8.83$ \\
\hline & 15 & $20.59 \pm 8.97$ & $19.76 \pm 4.76$ & $24.17 \pm 4.98$ & $21.99 \pm 1.33$ & $25.92 \pm 2.10$ \\
\hline & 20 & $12.64 \pm 4.89$ & $11.98 \pm 3.57$ & $14.04 \pm 3.01$ & $12.04 \pm 2.21$ & $17.11 \pm 2.18$ \\
\hline & 25 & $16.54 \pm 1.59$ & $14.09 \pm 2.95$ & $18.61 \pm 2.82$ & $6.92 \pm 1.04$ & $7.98 \pm 1.13$ \\
\hline \multirow{5}{*}{$30 \%$} & 5 & $8.99 \pm 2.07$ & $5.82 \pm 1.64$ & $12.09 \pm 3.18$ & $6.16 \pm 2.14$ & $8.95 \pm 2.03$ \\
\hline & 10 & $30.08 \pm 3.03$ & $28.76 \pm 3.54$ & $32.96 \pm 12.75$ & $31.85 \pm 11.85$ & $29.74 \pm 9.47$ \\
\hline & 15 & $25.98 \pm 6.73$ & $17.67 \pm 3.64$ & $26.82 \pm 3.92$ & $25.91 \pm 3.10$ & $21.81 \pm 0.99$ \\
\hline & 20 & $18.30 \pm 7.54$ & $14.89 \pm 3.76$ & $3.59 \pm 0.91$ & $18.73 \pm 1.99$ & $10.83 \pm 3.19$ \\
\hline & 25 & $9.45 \pm 3.09$ & $5.91 \pm 1.65$ & $19.73 \pm 5.03$ & $9.74 \pm 2.18$ & $15.98 \pm 1.96$ \\
\hline
\end{tabular}

TABLE 3: Lignin peroxidase (LiP) activity ( $\mathrm{Ug}^{-1} \mathrm{SMS}$ wet wt.) profiles of Coprinus cinereus at different stages during cultivation on solid sisal wastes supplemented with cow dung manure.

\begin{tabular}{|c|c|c|c|c|c|c|}
\hline \multirow{2}{*}{ Supplement level } & \multirow{2}{*}{ Days of incubation } & \multicolumn{5}{|c|}{ Substrate formulations } \\
\hline & & SL: SB $(100: 0)$ & SL: SB $(25: 75)$ & SL : SB $(50: 50)$ & SL: SB $(75: 25)$ & SL: SB $(0: 100)$ \\
\hline \multirow{5}{*}{$0 \%$} & 5 & $0.59 \pm 0.13$ & $0.34 \pm 0.09$ & $0.53 \pm 0.23$ & $0.27 \pm 0.11$ & $0.37 \pm 0.13$ \\
\hline & 10 & $1.93 \pm 0.62$ & $0.98 \pm 0.21$ & $1.48 \pm 0.17$ & $0.89 \pm 0.10$ & $0.67 \pm 0.22$ \\
\hline & 15 & $0.78 \pm 0.20$ & $1.07 \pm 0.30$ & $1.07 \pm 0.28$ & $1.06 \pm 0.27$ & $0.89 \pm 0.17$ \\
\hline & 20 & $0.91 \pm 0.11$ & $0.72 \pm 0.17$ & $0.34 \pm 0.10$ & $0.45 \pm 0.19$ & $0.53 \pm 0.10$ \\
\hline & 25 & $0.43 \pm 0.18$ & $0.27 \pm 0.12$ & $0.41 \pm 0.08$ & $0.53 \pm 0.21$ & $0.35 \pm 0.14$ \\
\hline \multirow{5}{*}{$10 \%$} & 5 & $0.68 \pm 0.24$ & $0.49 \pm 0.15$ & $0.34 \pm 0.07$ & $0.58 \pm 0.22$ & $0.47 \pm 0.12$ \\
\hline & 10 & $1.21 \pm 0.40$ & $1.04 \pm 0.28$ & $1.32 \pm 0.34$ & $1.10 \pm 0.43$ & $0.84 \pm 0.17$ \\
\hline & 15 & $0.99 \pm 0.19$ & $0.65 \pm 0.18$ & $1.01 \pm 0.23$ & $0.98 \pm 0.29$ & $0.61 \pm 0.20$ \\
\hline & 20 & $1.04 \pm 0.30$ & $0.74 \pm 0.20$ & $0.67 \pm 0.17$ & $0.49 \pm 0.10$ & $0.73 \pm 0.09$ \\
\hline & 25 & $0.67 \pm 0.15$ & $0.41 \pm 0.12$ & $0.48 \pm 0.12$ & $0.31 \pm 0.07$ & $0.46 \pm 0.17$ \\
\hline \multirow{5}{*}{$20 \%$} & 5 & $0.48 \pm 0.16$ & $0.57 \pm 0.24$ & $0.41 \pm 0.10$ & $0.42 \pm 0.06$ & $0.53 \pm 0.14$ \\
\hline & 10 & $1.09 \pm 0.20$ & $1.17 \pm 0.47$ & $1.12 \pm 0.33$ & $0.85 \pm 0.15$ & $1.04 \pm 0.30$ \\
\hline & 15 & $0.85 \pm 0.34$ & $0.87 \pm 0.23$ & $0.78 \pm 0.14$ & $1.03 \pm 0.43$ & $0.67 \pm 0.21$ \\
\hline & 20 & $0.97 \pm 0.26$ & $0.93 \pm 0.10$ & $0.85 \pm 0.25$ & $0.67 \pm 0.11$ & $0.71 \pm 0.13$ \\
\hline & 25 & $0.38 \pm 0.10$ & $0.27 \pm 0.14$ & $0.53 \pm 0.12$ & $0.46 \pm 0.18$ & $0.56 \pm 0.18$ \\
\hline \multirow{5}{*}{$30 \%$} & 5 & $0.61 \pm 0.12$ & $0.53 \pm 0.17$ & $0.44 \pm 0.16$ & $0.50 \pm 0.20$ & $0.40 \pm 0.14$ \\
\hline & 10 & $0.89 \pm 0.19$ & $1.07 \pm 0.43$ & $1.64 \pm 0.48$ & $1.13 \pm 0.38$ & $1.22 \pm 0.20$ \\
\hline & 15 & $1.09 \pm 0.32$ & $0.81 \pm 0.32$ & $0.89 \pm 0.29$ & $0.74 \pm 0.13$ & $0.78 \pm 0.11$ \\
\hline & 20 & $0.72 \pm 0.29$ & $0.56 \pm 0.11$ & $0.97 \pm 0.19$ & $0.87 \pm 0.10$ & $0.81 \pm 0.29$ \\
\hline & 25 & $0.49 \pm 0.14$ & $0.32 \pm 0.10$ & $0.57 \pm 0.13$ & $0.49 \pm 0.17$ & $0.36 \pm 0.10$ \\
\hline
\end{tabular}


TABLE 4: Carboxymethyl cellulase (CMCase) activity ( $\mathrm{Ug}^{-1}$ SMS wet wt.) profiles of Coprinus cinereus at different stages during cultivation on solid sisal wastes supplemented with cow dung manure.

\begin{tabular}{|c|c|c|c|c|c|c|}
\hline \multirow{2}{*}{ Supplement level } & \multirow{2}{*}{ Days of incubation } & \multicolumn{5}{|c|}{ Substrate formulations } \\
\hline & & SL: SB (100:0) & SL: SB $(25: 75)$ & SL: SB $(50: 50)$ & SL: SB $(75: 25)$ & SL : SB $(0: 100)$ \\
\hline \multirow{5}{*}{$0 \%$} & 5 & $0.67 \pm 0.07$ & $0.47 \pm 0.12$ & $0.19 \pm 0.08$ & $0.26 \pm 0.08$ & $0.39 \pm 0.10$ \\
\hline & 10 & $1.29 \pm 0.13$ & $0.35 \pm 0.12$ & $0.49 \pm 0.10$ & $0.40 \pm 0.13$ & $1.02 \pm 0.40$ \\
\hline & 15 & $0.80 \pm 0.03$ & $0.79 \pm 0.28$ & $0.71 \pm 0.15$ & $0.81 \pm 0.21$ & $1.35 \pm 0.08$ \\
\hline & 20 & $0.36 \pm 0.10$ & $0.95 \pm 0.20$ & $0.40 \pm 0.09$ & $1.48 \pm 0.40$ & $0.68 \pm 0.20$ \\
\hline & 25 & $0.26 \pm 0.09$ & $0.19 \pm 0.06$ & $0.55 \pm 0.15$ & $0.29 \pm 0.09$ & $0.34 \pm 0.11$ \\
\hline \multirow{5}{*}{$10 \%$} & 5 & $0.34 \pm 0.10$ & $0.44 \pm 0.10$ & $0.24 \pm 0.08$ & $0.33 \pm 0.12$ & $0.47 \pm 0.15$ \\
\hline & 10 & $0.92 \pm 0.20$ & $0.59 \pm 0.20$ & $0.58 \pm 0.18$ & $0.67 \pm 0.25$ & $0.45 \pm 0.10$ \\
\hline & 15 & $1.09 \pm 0.09$ & $0.78 \pm 0.13$ & $0.98 \pm 0.20$ & $0.91 \pm 0.04$ & $1.67 \pm 0.20$ \\
\hline & 20 & $0.27 \pm 0.05$ & $0.40 \pm 0.12$ & $0.32 \pm 0.10$ & $0.34 \pm 0.07$ & $1.23 \pm 0.30$ \\
\hline & 25 & $0.22 \pm 0.12$ & $0.18 \pm 0.06$ & $0.51 \pm 0.14$ & $0.28 \pm 0.12$ & $0.48 \pm 0.09$ \\
\hline \multirow{5}{*}{$20 \%$} & 5 & $0.29 \pm 0.08$ & $0.59 \pm 0.17$ & $0.35 \pm 0.10$ & $0.48 \pm 0.20$ & $0.41 \pm 0.20$ \\
\hline & 10 & $0.88 \pm 0.03$ & $0.75 \pm 0.05$ & $0.50 \pm 0.09$ & $0.35 \pm 0.10$ & $1.53 \pm 0.30$ \\
\hline & 15 & $0.93 \pm 0.28$ & $1.17 \pm 0.30$ & $0.86 \pm 0.11$ & $0.96 \pm 0.12$ & $2.03 \pm 0.70$ \\
\hline & 20 & $0.62 \pm 0.19$ & $0.30 \pm 0.09$ & $0.33 \pm 0.12$ & $1.65 \pm 0.30$ & $0.57 \pm 0.15$ \\
\hline & 25 & $0.18 \pm 0.07$ & $0.32 \pm 0.10$ & $0.17 \pm 0.02$ & $0.24 \pm 0.06$ & $0.36 \pm 0.10$ \\
\hline \multirow{5}{*}{$30 \%$} & 5 & $0.64 \pm 0.23$ & $0.39 \pm 0.08$ & $0.20 \pm 0.09$ & $0.56 \pm 0.15$ & $0.37 \pm 0.12$ \\
\hline & 10 & $0.72 \pm 0.05$ & $0.72 \pm 0.12$ & $0.69 \pm 0.26$ & $1.43 \pm 0.30$ & $0.97 \pm 0.20$ \\
\hline & 15 & $1.37 \pm 0.47$ & $1.04 \pm 0.20$ & $0.48 \pm 0.02$ & $2.83 \pm 1.12$ & $1.13 \pm 0.40$ \\
\hline & 20 & $1.06 \pm 0.30$ & $0.20 \pm 0.04$ & $0.10 \pm 0.03$ & $0.42 \pm 0.07$ & $0.68 \pm 0.20$ \\
\hline & 25 & $0.49 \pm 0.07$ & $0.28 \pm 0.09$ & $0.45 \pm 0.11$ & $0.22 \pm 0.09$ & $0.16 \pm 0.06$ \\
\hline
\end{tabular}

TABLE 5: Pectinase activity ( $\mathrm{Ug}^{-1}$ SMS wet wt.) profiles of Coprinus cinereus at different stages during cultivation on solid sisal wastes supplemented with cow dung manure.

\begin{tabular}{|c|c|c|c|c|c|c|}
\hline \multirow{2}{*}{ Supplement level } & \multirow{2}{*}{ Days of incubation } & \multicolumn{5}{|c|}{ Substrate formulations } \\
\hline & & SL: SB $(100: 0)$ & SL : SB $(25: 75)$ & SL : SB $(50: 50)$ & SL: SB $(75: 25)$ & SL : SB $(0: 100)$ \\
\hline \multirow{5}{*}{$0 \%$} & 5 & $0.13 \pm 0.05$ & $0.08 \pm 0.03$ & $0.19 \pm 0.08$ & $0.25 \pm 0.10$ & $0.36 \pm 0.10$ \\
\hline & 10 & $0.36 \pm 0.11$ & $0.26 \pm 0.12$ & $0.13 \pm 0.05$ & $0.59 \pm 0.18$ & $0.49 \pm 0.14$ \\
\hline & 15 & $0.76 \pm 0.14$ & $0.50 \pm 0.12$ & $0.36 \pm 0.08$ & $0.88 \pm 0.10$ & $1.16 \pm 0.15$ \\
\hline & 20 & $0.97 \pm 0.20$ & $0.95 \pm 0.11$ & $0.60 \pm 0.15$ & $0.87 \pm 0.22$ & $0.46 \pm 0.10$ \\
\hline & 25 & $1.46 \pm 0.13$ & $0.84 \pm 0.05$ & $0.79 \pm 0.28$ & $1.59 \pm 0.40$ & $1.12 \pm 0.27$ \\
\hline \multirow{5}{*}{$10 \%$} & 5 & $0.23 \pm 0.08$ & $0.13 \pm 0.05$ & $0.11 \pm 0.04$ & $0.19 \pm 0.09$ & $0.27 \pm 0.12$ \\
\hline & 10 & $0.74 \pm 0.30$ & $0.37 \pm 0.13$ & $0.26 \pm 0.10$ & $0.47 \pm 0.16$ & $0.51 \pm 0.20$ \\
\hline & 15 & $1.32 \pm 0.13$ & $0.56 \pm 0.10$ & $0.47 \pm 0.18$ & $1.28 \pm 0.21$ & $1.32 \pm 0.22$ \\
\hline & 20 & $1.05 \pm 0.24$ & $0.72 \pm 0.14$ & $0.86 \pm 0.10$ & $0.66 \pm 0.09$ & $0.28 \pm 0.08$ \\
\hline & 25 & $1.90 \pm 0.32$ & $0.79 \pm 0.15$ & $0.59 \pm 0.16$ & $0.91 \pm 0.17$ & $0.57 \pm 0.18$ \\
\hline \multirow{5}{*}{$20 \%$} & 5 & $0.18 \pm 0.07$ & $0.22 \pm 0.09$ & $0.18 \pm 0.07$ & $0.09 \pm 0.02$ & $0.19 \pm 0.09$ \\
\hline & 10 & $0.57 \pm 0.20$ & $0.47 \pm 0.14$ & $0.31 \pm 0.10$ & $0.57 \pm 0.12$ & $0.47 \pm 0.16$ \\
\hline & 15 & $1.10 \pm 0.23$ & $0.59 \pm 0.17$ & $0.59 \pm 0.14$ & $1.52 \pm 0.37$ & $1.81 \pm 0.14$ \\
\hline & 20 & $0.71 \pm 0.10$ & $0.31 \pm 0.07$ & $0.77 \pm 0.18$ & $0.74 \pm 0.10$ & $0.38 \pm 0.12$ \\
\hline & 25 & $0.22 \pm 0.08$ & $1.43 \pm 0.18$ & $0.99 \pm 0.30$ & $1.08 \pm 0.30$ & $0.64 \pm 0.10$ \\
\hline \multirow{5}{*}{$30 \%$} & 5 & $0.21 \pm 0.08$ & $0.27 \pm 0.11$ & $0.20 \pm 0.09$ & $0.31 \pm 0.14$ & $0.29 \pm 0.12$ \\
\hline & 10 & $0.75 \pm 0.30$ & $0.39 \pm 0.10$ & $0.25 \pm 0.13$ & $0.53 \pm 0.20$ & $0.68 \pm 0.20$ \\
\hline & 15 & $1.22 \pm 0.16$ & $0.92 \pm 0.03$ & $0.42 \pm 0.08$ & $1.12 \pm 0.18$ & $1.77 \pm 0.11$ \\
\hline & 20 & $0.98 \pm 0.20$ & $0.66 \pm 0.20$ & $0.84 \pm 0.12$ & $0.63 \pm 0.04$ & $0.63 \pm 0.10$ \\
\hline & 25 & $1.79 \pm 0.50$ & $1.51 \pm 0.11$ & $0.58 \pm 0.15$ & $1.16 \pm 0.28$ & $0.40 \pm 0.07$ \\
\hline
\end{tabular}


TABLE 6: Xylanase activity ( $\mathrm{Ug}^{-1} \mathrm{SMS}$ wet wt.) profiles of Coprinus cinereus at different stages during cultivation on solid sisal wastes supplemented with cow dung manure.

\begin{tabular}{|c|c|c|c|c|c|c|}
\hline \multirow{2}{*}{ Supplement level } & \multirow{2}{*}{ Days of incubation } & \multicolumn{5}{|c|}{ Substrate formulations } \\
\hline & & SL: SB $(100: 0)$ & SL : SB $(25: 75)$ & SL: SB $(50: 50)$ & SL: SB $(75: 25)$ & SL: SB $(0: 100)$ \\
\hline \multirow{5}{*}{$0 \%$} & 5 & $0.09 \pm 0.04$ & $0.27 \pm 0.09$ & $0.17 \pm 0.09$ & $0.24 \pm 0.10$ & $0.15 \pm 0.07$ \\
\hline & 10 & $0.58 \pm 0.10$ & $0.45 \pm 0.14$ & $0.37 \pm 0.14$ & $0.44 \pm 0.14$ & $0.33 \pm 0.11$ \\
\hline & 15 & $0.79 \pm 0.20$ & $0.76 \pm 0.12$ & $0.97 \pm 0.10$ & $0.73 \pm 0.12$ & $1.06 \pm 0.24$ \\
\hline & 20 & $0.21 \pm 0.09$ & $0.61 \pm 0.10$ & $0.87 \pm 0.30$ & $0.59 \pm 0.06$ & $0.66 \pm 0.16$ \\
\hline & 25 & $0.15 \pm 0.06$ & $0.30 \pm 0.18$ & $0.41 \pm 0.07$ & $0.51 \pm 0.09$ & $0.31 \pm 0.12$ \\
\hline \multirow{5}{*}{$10 \%$} & 5 & $0.15 \pm 0.06$ & $0.31 \pm 0.07$ & $0.27 \pm 0.11$ & $0.26 \pm 0.08$ & $0.18 \pm 0.07$ \\
\hline & 10 & $0.27 \pm 0.11$ & $0.63 \pm 0.20$ & $0.43 \pm 0.13$ & $0.33 \pm 0.10$ & $0.24 \pm 0.09$ \\
\hline & 15 & $0.39 \pm 0.06$ & $1.23 \pm 0.12$ & $0.81 \pm 0.07$ & $0.98 \pm 0.14$ & $0.70 \pm 0.20$ \\
\hline & 20 & $0.18 \pm 0.07$ & $0.62 \pm 0.09$ & $0.96 \pm 0.20$ & $0.60 \pm 0.15$ & $0.48 \pm 0.14$ \\
\hline & 25 & $0.10 \pm 0.04$ & $0.47 \pm 0.10$ & $0.56 \pm 0.06$ & $0.25 \pm 0.07$ & $0.33 \pm 0.10$ \\
\hline \multirow{5}{*}{$20 \%$} & 5 & $0.24 \pm 0.09$ & $0.43 \pm 0.10$ & $0.16 \pm 0.05$ & $0.19 \pm 0.07$ & $0.15 \pm 0.06$ \\
\hline & 10 & $0.67 \pm 0.12$ & $0.72 \pm 0.22$ & $1.09 \pm 0.04$ & $0.27 \pm 0.11$ & $0.50 \pm 0.18$ \\
\hline & 15 & $0.95 \pm 0.20$ & $0.97 \pm 0.18$ & $0.92 \pm 0.16$ & $0.79 \pm 0.15$ & $0.89 \pm 0.10$ \\
\hline & 20 & $0.39 \pm 0.06$ & $0.66 \pm 0.09$ & $0.49 \pm 0.09$ & $0.61 \pm 0.09$ & $0.55 \pm 0.19$ \\
\hline & 25 & $0.14 \pm 0.05$ & $0.44 \pm 0.14$ & $0.42 \pm 0.12$ & $0.40 \pm 0.07$ & $0.16 \pm 0.04$ \\
\hline \multirow{5}{*}{$30 \%$} & 5 & $0.39 \pm 0.10$ & $0.51 \pm 0.16$ & $0.23 \pm 0.09$ & $0.30 \pm 0.10$ & $0.19 \pm 0.08$ \\
\hline & 10 & $0.47 \pm 0.13$ & $0.67 \pm 0.23$ & $0.36 \pm 0.14$ & $0.58 \pm 0.19$ & $0.32 \pm 0.10$ \\
\hline & 15 & $1.04 \pm 0.20$ & $0.99 \pm 0.07$ & $0.94 \pm 0.20$ & $0.89 \pm 0.08$ & $0.88 \pm 0.13$ \\
\hline & 20 & $0.76 \pm 0.16$ & $0.70 \pm 0.15$ & $0.63 \pm 0.15$ & $0.45 \pm 0.11$ & $0.45 \pm 0.15$ \\
\hline & 25 & $0.31 \pm 0.07$ & $0.25 \pm 0.12$ & $0.41 \pm 0.08$ & $0.23 \pm 0.07$ & $0.36 \pm 0.18$ \\
\hline
\end{tabular}

either second or third flush. The highest pectinase activity of $1.90 \pm 0.32 \mathrm{Ug}^{-1}$ was revealed after the third flush harvest on $10 \%$ supplemented SL : SB $(0: 100)$ substrate formulation.

3.2.5. Xylanase Enzymes ( $U g^{-1} S M S$ Wet $W t$.). The xylanase activity varied with substrate formulation and supplementation rate during developmental stages. The results showed that xylanase activity was increasing as incubation days increase. It was found to be higher either during full mycelia colonization or after the first flush harvest; besides, the rate of xylanase production was slightly increased in fruiting phase compared with mycelia growth period. The highest activity of $1.23 \pm 0.12 \mathrm{Ug}^{-1}$ was observed on $10 \%$ supplemented SL : SB $(25: 75)$ after the first flush harvest (Table 6).

\section{Discussion}

4.1. Spawn Run and Pinhead and Fruiting Bodies Formation. The present study revealed that the efficient colonization and utilization by $C$. cinereus varied among solid sisal waste formulations with or without addition of supplement, which depended upon the ability of this fungus to produce extracellular enzymes required to degrade major components of the sisal waste biomass. It means that $C$. cinereus are capable of degrading lignocellulosic polysaccharides to ensure mycelia with carbon and energy source for extensive substrate colonization and fruit body formation [1]. In addition, not only the enzymes profiles but also their physicochemical characteristics when they are secreted account for the capacity of colonization and degradation; more stable enzymes at their optimum $\mathrm{pH}$ activity are capable of producing more extensive degradation of the substrate [19]. The number of days taken for completion of spawn run and those for pinheads formation differed significantly $(p<0.05)$ indicating the difference in composition among the substrate formulations with or without supplement. These results are in agreement with previously reported findings. Gaitán-Hernández et al. [20] did correlation studies between the constituents of the substrates and the number of days to primordium formation; they discovered a significant positive relationship with cellulose content and total sugar indicating that cellulose and sugar content in each substrate were directly proportional to the spawning duration and the primordium formation. Similarly, Oei [21] reported that substrate having high quality lignin and cellulose contents takes a longer time to start pinning and fruit body formation.

4.2. Enzyme Profile during Solid State Cultivation of Coprinus cinereus on Sisal Waste Formulations. Many fungal species have the ability to degrade lignocellulosic biomass by producing extracellular enzymes. The extracellular enzymes production by solid state fermentation plays a crucial role supplying carbon and energy source consequently providing the fungus with the material for biosynthetic activity. The present study focuses on measuring two oxidative (laccase and lignin peroxidase) and three hydrolytic (carboxymethyl cellulase, pectinase, and xylanase) enzymes secreted by Coprinus cinereus, using sisal waste fractions amended with cow manure. In addition, the study shows the profiles of 
extracellular enzymatic activities produced during different cultivation stages varied among the sisal waste substrate formulations supplemented with cow manure. The differences found in enzyme production might be due to the relative composition of polysaccharides, the size of the wastes used, and probably the presence of natural inducers such as aromatic compounds [22]. In general, the yields of lignocellulolytic enzymes were expected to increase as supplementation rate increases but this was not the case in this study. This could be due to repression effect caused by addition of cow manure [23]. Moreover, in the literature, contradictory evidence exists for the effects of the nature and concentration of the nitrogen source on ligninolytic enzyme production. While high nitrogen media gave the highest laccase activity in Lentinus edodes, Rigidoporus lignosus, and Trametes pubescens, nitrogen-limited conditions enhanced the production of the enzyme in Pycnoporus cinnabarinus, $P$. sanguineus, and Phlebia radiata $[24,25]$. The role of these compounds in the regulation of enzyme synthesis depends not only on the physiology of the tested fungi but also on the medium composition, especially on the presence of lignocellulosic substrate [26, 27]. Of the lignocellulolytic enzymes assayed, laccase was detected in the highest quantity, in terms of total units per substrate.

Laccase was actively secreted during mycelia run and reached the maximum level during full mycelia colonization and then declined gradually during fruiting. The laccase increased again after harvesting either first or second flushes while in lignin peroxidase (LiP) a wave-like pattern was observed, with the highest peaks found during full mycelia colonization or soon after first flush harvest. Higher levels of laccase activity during early stage of the spawn run indicate that this enzyme is responsible for degradation of physical barrier of lignin so that more cellulosic and hemicellulosic content is exposed for further degradation to extract the energy for the growth of mushroom fungus [28]. It has also been well documented that composition of the substrate and the method of cultivation influence the pattern of enzyme production by white rot fungi $[29,30]$. The present findings are similar as reported by several authors working with different basidiomycetes [30-33]. The study of Periasamy [34] using Pleurotus djamor var. roseus correlated the increase of laccase activity to growth and differentiation of fruiting bodies and decreasing levels of lignin content. Moreover, Thurston [35] showed that white rot fungi laccase, besides functioning as the lignin degrading enzyme, was also important in pigment production, polyphenol detoxification, fruiting body formation, and sporulation and as antimicrobial agent. In general, ligninolytic enzyme increased at somatic phase and decreased at the fruiting body phase. However, it is assumed that when the fruiting body was reformed at the next incubation, the fluctuation of ligninolytic enzyme activity occurred.

With respect to hydrolytic enzymes, CMCase enzyme activity gradually increased coinciding with fungal growth and maximum peaks were detected after either first flush or second flush (Table 4), suggesting a role in the morphogenesis of fruiting bodies as well as a function in overall nutrition of this mushroom species [36]. Sharma and Arora [37] associated elevated CMCase levels produced by mycelia on lignocellulosic substrates with high biological efficiencies.

Pectinase activity showed a gradual increase during mycelial growth and a sharp increase during fructification in all substrate formulations. A common feature among pectic enzymes that could be responsible for this increase of pectinase activity is their glucose repressible synthesis [38]. The results of xylanase production show a positive correlation with those obtained by Isikhuemhen and Mikiashvilli [39], who observed higher xylanase productivity from Pleurotus ostreatus in fruiting than mycelial growth period when cultivated on some solid wastes. Similarly, Terashita et al. [40] observed xylanase activity increasing during vegetative mycelial growth and maximum activity was reached after cropping of $P$. flabellatus and $P$. sajor-caju. The low activity of xylanase during the beginning of incubation could probably be due to the fact that xylanase was repressed by the ligninolytic enzyme, which was active in the beginning as reported by Singh et al. [28]. The increase in xylanase activity beyond full mycelia colonization is an indication that the enzyme was active in hydrolyzing hemicellulose during this stage. This also suggests that hemicellulose is not utilized in the beginning by the growing mycelium. However, a contrary result was found by Chen et al. [41] who reported that xylanolytic activity was crucial for the vegetative growth of fungus. Ahlawat et al. [42] also observed that xylanase was at peak on the 10th day during cultivation of Volvariella volvacea on paddy straw and was crucial during vegetative growth of the fungus.

\section{Conclusion}

In the present work, it was shown that Coprinus cinereus can be successfully cultivated on solid sisal waste formulations amended with cow dung manure probably because they produce enzymatic activities that are necessary for lignocellulose degradation, as well as for developmental regulation. The pattern of both hydrolytic and oxidative activity of $C$. cinereus enzymes was probably affected by the solid sisal waste substrates, growth stage, and the development of mushroom. The investigation of the hydrolytic and oxidative enzymes production during edible mushroom cultivation is required because after harvesting of fruit bodies the residual spent substrate may become a cheap source of lignocellulolytic enzymes for several applications including bioremediation and enzymes production as shown above.

\section{Conflict of Interests}

The authors declare that there is no conflict of interests regarding the publication of this paper.

\section{Acknowledgment}

The authors wish to acknowledge the World Bank project CIA 3.3 component "Industrial Bioconversion of Selected Tanzanian Crops and Residues into Value Added Products Using Biotechnology" at the Department of Molecular Biology 
and Biotechnology (DMBB), College of Natural and Applied Sciences (CoNAS), University of Dar es Salaam (UDSM), for financial support.

\section{References}

[1] J. A. Buswell, Y. J. Cai, S. T. Chang, J. F. Peberdy, S. Y. Fu, and H.S. Yu, "Lignocellulolytic enzyme profiles of edible mushroom fungi," World Journal of Microbiology and Biotechnology, vol. 12, no. 5, pp. 537-542, 1996.

[2] A. M. Mshandete, L. Björnsson, A. K. Kivaisi, M. S. T. Rubindamayugi, and B. Mattiasson, "Two-stage anaerobic digestion of aerobic pre-treated sisal leaf decortications residues: hydrolases activities and biogas production profile," African Journal of Biochemistry Research, vol. 2, no. 11, pp. 211-218, 2008.

[3] M. Bonatti, P. Karnopp, H. M. Soares, and S. A. Furlan, "Evaluation of Pleurotus ostreatus and Pleurotus sajor-caju nutritional characteristics when cultivated in different lignocellulosic wastes," Food Chemistry, vol. 88, no. 3, pp. 425-428, 2004.

[4] A. M. Mshandete and J. Cuff, "Cultivation of three types of indigenous wild edible mushrooms: Coprinus cinereus, Pleurotus flabellatus and Volvariella volvocea on composted sisal decortications residue in Tanzania," African Journal of Biotechnology, vol. 7, no. 24, pp. 4551-4562, 2008.

[5] P. Raymond, A. M. Mshandete, and A. K. Kivaisi, "Comparative study on cultivation and yield performance of Coprinus cinereus (Schaeff) Gray on sisal wastes supplemented with cow dung manure," International Journal of Research in Pure and Applied Microbiology, vol. 2, no. 3, pp. 25-31, 2012.

[6] V. Elisashvili, M. Penninckx, E. Kachlishvili, M. Asatiani, and G. Kvesitadze, "Use of Pleurotus dryinus for lignocellulolytic enzymes production in submerged fermentation of mandarin peels and tree leaves," Enzyme and Microbial Technology, vol. 38, no. 7, pp. 998-1004, 2006.

[7] Y. H. Tan and M. N. Wahab, "Extracellular enzyme production during anamorphic growth in the edible mushroom, Pleurotus sajor-caju," World Journal of Microbiology and Biotechnology, vol. 13, no. 6, pp. 613-617, 1997.

[8] O. O. Kuforiji and I. O. Fasidi, "Enzyme activities of Pleurotus tuber-regium (Fries) Singer, cultivated on selected agricultural wastes," Bioresource Technology, vol. 99, no. 10, pp. 4275-4278, 2008.

[9] S. Kurt and S. Buyukalaca, "Yield performances and changes in enzyme activities of Pleurotus spp. (P. Ostreatus and P. SajorCaju) cultivated on different agricultural wastes," Bioresource Technology, vol. 101, no. 9, pp. 3164-3169, 2010.

[10] A. Mshandete, L. Björnsson, A. K. Kivaisi, M. S. T. Rubindamayugi, and B. Mattiasson, "Effect of particle size on biogas yield from sisal fibre waste," Renewable Energy, vol. 31, no. 14, pp. 2385-2392, 2006.

[11] M. Muthangya, A. Mshandete, and A. K. Kivaisi, "Enhancement of anaerobic digestion of sisal leaf decortication residues by biological pre-treatment," ARPN Journal of Agricultural and Biological Science, vol. 4, pp. 66-73, 2009.

[12] A. M. Mshandete, O. kibazohi, and A. K. Kivaisi, “Tanzania sisal industry: auditing and characterization of sisal post-harvest wastes as a bio-resource of bio-refining," International Journal of Pure and Applied Sciences and Technology, vol. 14, no. 2, pp. 84-94, 2013.

[13] A. Dhouib, M. Hamza, H. Zouari et al., "Autochthonous fungal strains with high ligninolytic activities from Tunisian biotopes," African Journal of Biotechnology, vol. 4, no. 5, pp. 431-436, 2005.
[14] H. Risdianto, S. H. Suhardi, T. Setiadi, and T. Kokugan, "The influence of temperature on laccase production in solid state fermentation by using white rot fungus Marasmius sp," in Proceedings of the 1st International Seminar on Fundamental and Application of Chemical Engineering, Bali, Indonesia, November 2010.

[15] F. Patrick, G. Y. S. Mtui, A. M. Mshandete, and A. K. Kivaisi, "Ligninolytic enzymes activities of Pleurotus sapidus P969 during vegetative growth and fruit development on sugarcane residues-based substrates," International Journal of Biotechnology, vol. 3, no. 4, pp. 58-71, 2014.

[16] M. Sugiura, H. Hirai, and T. Nishida, "Purification and characterization of a novel lignin peroxidase from white-rot fungus Phanerochaete sordida YK-624," FEMS Microbiology Letters, vol. 224, no. 2, pp. 285-290, 2003.

[17] G. L. Miller, "Use of dinitrosalicylic acid reagent for determination of reducing sugar," Analytical Chemistry, vol. 31, no. 3, pp. 426-428, 1959.

[18] T. K. Ghose, "Measurement of cellulase activities," Pure and Applied Chemistry, vol. 59, no. 2, pp. 257-268, 1987.

[19] B. E. Lechner and V. L. Papinutti, "Production of lignocellulosic enzymes during growth and fruiting of the edible fungus Lentinus tigrinus on wheat straw," Process Biochemistry, vol. 41, no. 3, pp. 594-598, 2006.

[20] R. Gaitán-Hernández, M. Esqueda, A. Gutiérrez, and M. Beltrán-García, "Quantitative changes in the biochemical composition of lignocellulosic residues during the vegetative growth of Lentinula edodes," Brazilian Journal of Microbiology, vol. 42, no. 1, pp. 30-40, 2011.

[21] P. Oei, Mushroom Cultivation. Appropriate Technology for Mushroom Grawres, Backhuys Publisher, Leiden, The Netherlands, 3rd edition, 2003.

[22] V. L. Papinutti and F. Forchiassin, "Lignocellulolytic enzymes from Fomes sclerodermeus growing in solid-state fermentation," Journal of Food Engineering, vol. 81, no. 1, pp. 54-59, 2007.

[23] N. Mikiashvili, V. Elisashvili, S. Wasser, and E. Nevo, "Carbon and nitrogen sources influence the ligninolytic enzyme activity of Trametes versicolor," Biotechnology Letters, vol. 27, no. 13, pp. 955-959, 2005.

[24] L. Gianfreda, F. Xu, and J.-M. Bollag, "Laccases: a useful group of oxidoreductive enzymes," Bioremediation Journal, vol. 3, no. 1, pp. 1-25, 1999.

[25] C. Galhaup, H. Wagner, B. Hinterstoisser, and D. Haltrich, "Increased production of laccase by the wood-degrading basidiomycete Trametes pubescens," Enzyme and Microbial Technology, vol. 30, no. 4, pp. 529-536, 2002.

[26] S. R. Couto, E. Rosales, M. Gundín, and M. Á. Sanromán, "Exploitation of a waste from the brewing industry for laccase production by two Trametes species," Journal of Food Engineering, vol. 64, no. 4, pp. 423-428, 2004.

[27] A. N. Kapich, B. A. Prior, A. Botha, S. Galkin, T. Lundell, and A. Hatakka, "Effect of lignocellulose-containing substrates on production of ligninolytic peroxidases in submerged cultures of Phanerochaete chrysosporium ME-446," Enzyme and Microbial Technology, vol. 34, no. 2, pp. 187-195, 2004.

[28] C. S. Singh, V. K. Singh, S. P. Tiwari, and R. Sharma, "Lignolytic activity and lignocellulosic degradation profiles by natural isolates of Pleurotus flabellatus during mushroom cultivation on rice straw," Asian Journal of Experimental Biological Sciences, vol. 2, no. 1, pp. 158-161, 2011.

[29] V. L. Papinutti, L. A. Diorio, and F. Forchiassin, "Production of laccase and manganese peroxidase by Fomes sclerodermeus 
grown on wheat bran," Journal of Industrial Microbiology and Biotechnology, vol. 30, no. 3, pp. 157-160, 2003.

[30] V. Elisashvili, M. Penninckx, E. Kachlishvili et al., "Lentinus edodes and Pleurotus species lignocellulolytic enzymes activity in submerged and solid-state fermentation of lignocellulosic wastes of different composition," Bioresource Technology, vol. 99, no. 3, pp. 457-462, 2008.

[31] M. Rühl, C. Fischer, and U. Kües, "Ligninolytic enzyme activities alternate with mushroom production during industrial cultivation of Pleurotus ostreatus on wheat straw-based substrate," Current Trends in Biotechnology and Pharmacy, vol. 2, no. 4, pp. 478-492, 2008.

[32] F. Patrick, G. Y. S. Mtui, A. M. Mshandete, and A. K. Kivaisi, "Ligninolytic enzymes activities of Pleurotus sapidus P969 during vegetative growth and fruit development on sugarcane residues-based substrates," The International Journal of Biotechnology, vol. 3, no. 4, pp. 58-71, 2014.

[33] M. Muthangya, A. M. Mshandete, S. O. Hashim, M. J. Amana, and A. K. Kivaisi, "Evaluation of enzymatic activity during vegetative growth and fruiting of Pleurotus HK 37 on Agave sisalana saline solid waste," Journal of Chemical, Biological and Physical Sciences, vol. 4, no. 1, pp. 247-258, 2014.

[34] K. Periasamy, Studies on Pleurotus djamor (Fr.) Boedijn var. roseus corner [Ph.D. thesis], University of Madras, Chennai, India, 2001.

[35] C. F. Thurston, "The structure and function of fungal laccases," Microbiology, vol. 140, no. 1, pp. 19-26, 1994.

[36] D. Geetha and K. Sivaprakasam, "Enzyme and sporophore production potential of oyster mushroom (Pleurotus spp)," Mushroom Research, vol. 7, pp. 39-42, 1998.

[37] R. K. Sharma and D. S. Arora, "Production of lignocellulolytic enzymes and enhancement of in vitro digestibility during solid state fermentation of wheat straw by Phlebia floridensis," Bioresource Technology, vol. 101, no. 23, pp. 9248-9253, 2010.

[38] P. Blanco, C. Sieiro, and T. G. Villa, "Production of pectic enzymes in yeasts," FEMS Microbiology Letters, vol. 175, no. 1, pp. 1-9, 1999.

[39] O. S. Isikhuemhen and N. A. Mikiashvilli, "Lignocellulolytic enzyme activity, substrate utilization, and mushroom yield by Pleurotus ostreatus cultivated on substrate containing anaerobic digester solids," Journal of Industrial Microbiology and Biotechnology, vol. 36, no. 11, pp. 1353-1362, 2009.

[40] T. Terashita, R. Murao, K. Yoshikawa, and J. Shishiyama, "Changes in carbohydrase activities during vegetative growth and development of fruit-bodies of Hypsizygus marmoreus grown in sawdust-based culture," Journal of Wood Science, vol. 44, no. 3, pp. 234-236, 1998.

[41] S. Chen, W. Ge, and J. A. Buswell, "Molecular cloning of a new laccase from the edible straw mushroom Volvariella volvacea: possible involvement in fruit body development," FEMS Microbiology Letters, vol. 230, no. 2, pp. 171-176, 2004.

[42] O. P. Ahlawat, K. Ahlawat, and B. L. Dhar, "Influence of lignocel lulolytic enzymes on substrate colonization and yield in monosporous isolates and parent's strains of Volvariella volvacea (Bull. Fr.) Sing," Indian Journal of Microbiology, vol. 45, no. 3, pp. 205-210, 2005. 

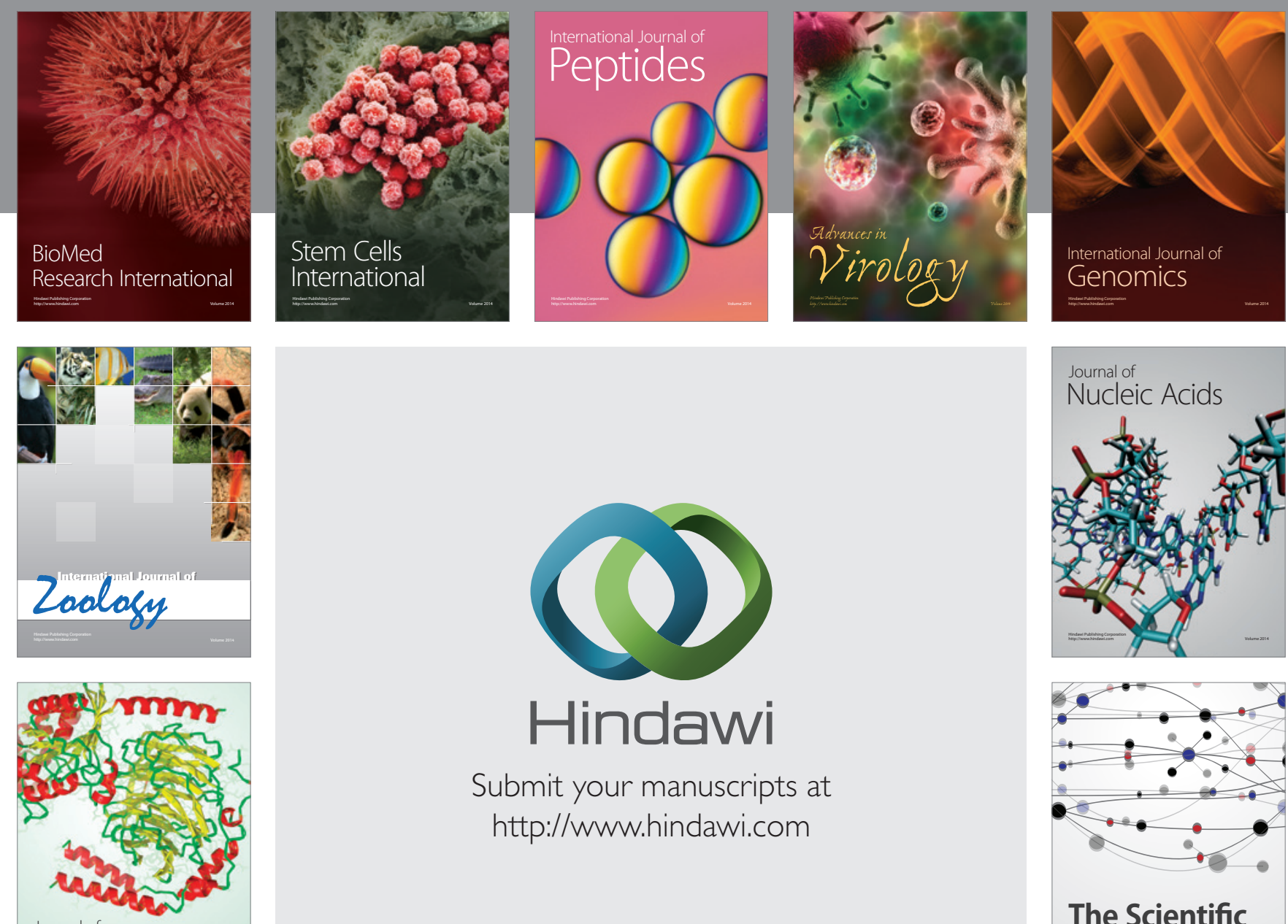

Submit your manuscripts at

http://www.hindawi.com

Journal of
Signal Transduction
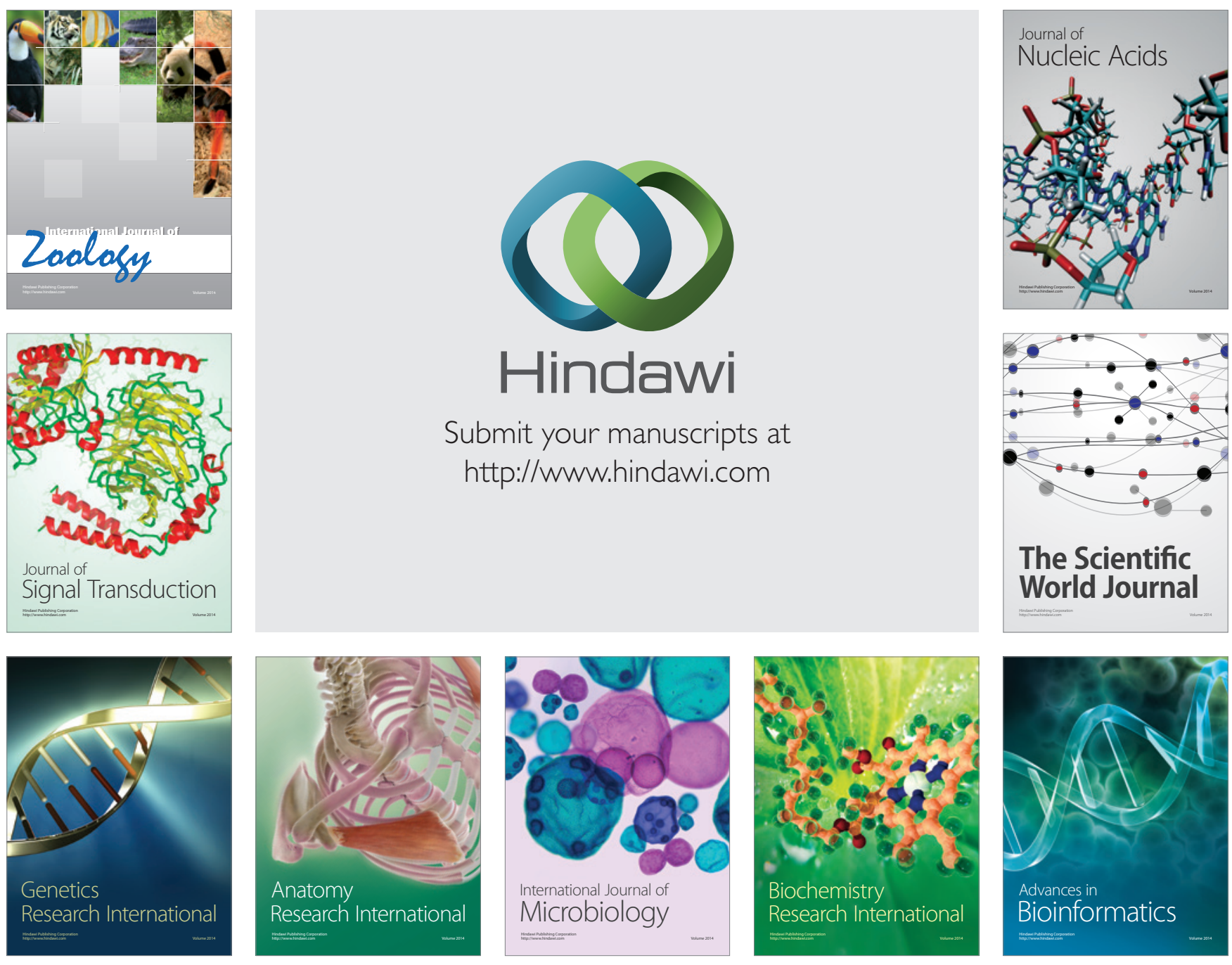

The Scientific World Journal
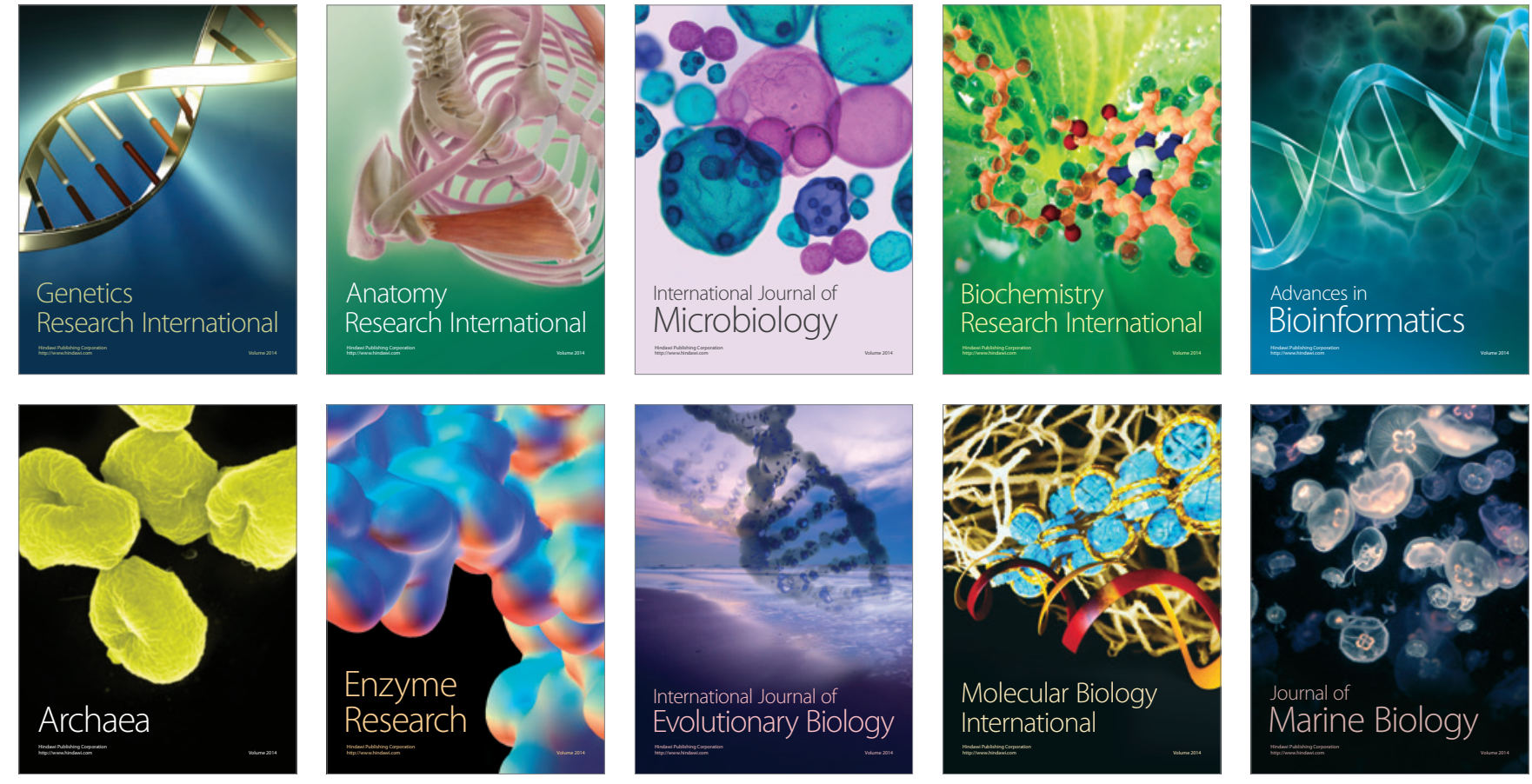